\title{
A simple method for the reduction of boundary effects in the Richardson-Lucy approach to image deconvolution
}

\author{
M. Bertero and P. Boccacci
}

\author{
DISI, Università di Genova, via Dodecaneso 35, 16146 Genova, Italy \\ e-mail: bertero@disi.unige.it
}

Received 18 January 2005 / Accepted 17 March 2005

\begin{abstract}
In this paper we propose a solution to the problem of reducing the boundary effects (ripples) in the deconvolution of astronomical images. The approach applies to the Richardson-Lucy method (RLM), namely the most frequently used deconvolution method in Astronomy, and is based on the idea of using RLM for attempting a reconstruction of the astronomical target in a domain broader than that of the detected image. Even if, in general, the reconstruction outside the image domain is not reliable, this approach, in a sense, is letting RLM to choose the appropriate boundary conditions and, as a consequence, the reconstruction inside the domain is considerably improved. We propose a simple implementation of this approach, allowing a reduction of its computational burden. Numerical experiments indicate that it is possible to obtain excellent results. Extensions and applications of the method are briefly discussed.
\end{abstract}

Key words. methods: data analysis - methods: numerical

\section{Introduction}

Deblurring is an important issue in the processing of astronomical images. If the observed object is in the center of the image domain and is surrounded by a region of free sky, with an angular size greater than the width of the point spread function (PSF), then FFT-based methods for image deconvolution can be successfully and efficiently used. RLM is the most popular one in Astronomy; it requires 4 FFTs per iteration, hence with a cost of $O\left(N^{2} \log _{2} N\right)$ operations in the case of $N \times N$ images.

However, as a consequence of the limited field of view (FOV) of the telescope, it may happen that an extended object is not completely contained within the image domain; in other words the boundaries of the image do not correspond to free sky. In such a case the standard FFT-based RLM can not be successfully used. Indeed, the use of the FFT implicitly assumes a periodic continuation of the image outside the original domain; as a consequence, discontinuities appear at the boundaries and, in the deconvolved image, these discontinuities generate Gibbs oscillations (sometimes called ripples), which can propagate inside the image domain and degrade completely the quality of the reconstruction.

Approaches for reducing these effects are based on the apodization of the detected image or on a change of the boundary conditions (BC). Reflective (or symmetric) BC are proposed by Chan et al. (1999), and anti-reflective BC are proposed by Serra Capizzano (2003). These conditions remove the discontinuities at the boundary. For instance, reflective BC preserve the continuity of the image while the anti-reflective ones preserve the continuity of the image and its gradient. In general, the use of $\mathrm{BC}$ provides a reduction of the ripples (sometimes by a factor 10). However we must point out that:

- the use of some specific BC is equivalent to extend the image outside the FOV by means of some symmetry rule; for instance, in the case of reflective $\mathrm{BC}$, the data outside the FOV are obtained by reflection of the data inside; such a procedure, in general, does not produce the correct values of the image and of the target outside the FOV;

- all the BC approaches have been implemented and tested in the framework of least-squares methods (LSM) even if they can be applied also to RLM;

- as far as we know, efficient implementation is possible only in the case of symmetric PSF, a condition which is hardly satisfied in astronomical imaging.

For these reasons we think that other ways of looking at the problem must be investigated. In particular the requirement of a symmetric PSF must be removed and, in fact, is not relevant in the method we propose in this paper.

The basic point is that, as a consequence of the finite extent of the PSF, values of the object outside the FOV significantly contribute to the values of the image in the pixels which are close to the boundary. Therefore one can formulate the problem by introducing these values as unknown parameters which must be estimated by the deconvolution method. The main difficulties generated by this approach are evident:

- the problem is under-determined because the number of unknown values is greater than the number of data; 
- the computational efficiency provided by FFT is lost since the problem can not be formulated as a standard deconvolution problem.

In this paper we propose a way for overcoming these difficulties in the case of RLM, even if the approach can be applied also to LSMs. In the case of RLM the computational cost per iteration is that corresponding to an image $2 N \times 2 N$ instead of $N \times N$, hence it is increased by a factor of about 16 . The method is very simple and can be easily implemented by astronomers interested in its use. Moreover, it can be easily extended to the case of multiple interferometric images, a problem of great interest for the future Large Binocular Telescope (LBT).

\section{The method}

We assume that the detected image $\boldsymbol{g}$ is an array $N \times N$. Due to the finite extent of the PSF, it receives contributions from a broader array $\boldsymbol{f}$, let us say $N^{\prime} \times N^{\prime}$, formed by the values of the unknown object. The value of $N^{\prime}>N$ depends on the extent of the PSF and, at this stage of our analysis, is not relevant. We also assume that the two arrays are indexed by a multiindex $\boldsymbol{n}=\left\{n_{1}, n_{2}\right\}$, which is defined in such a way that the same value is attributed to corresponding pixels in the two arrays. For instance, if $\{0,0\}$ is the bottom-left pixel of the object array, then the bottom-left pixel of $\boldsymbol{g}$ corresponds to $\left\{N^{\prime \prime}, N^{\prime \prime}\right\}$, with $N^{\prime \prime}=\left(N^{\prime}-N\right) / 2$ (both $N$ and $N^{\prime}$ are even). We denote by $S^{\prime}$ the set of the values of the multi-index $\boldsymbol{n}$ corresponding to the object, whence with $n_{1}, n_{2}$ varying from 0 to $N^{\prime}-1$, and by $S$ the set of the values corresponding to the image, hence with $n_{1}, n_{2}$ varying from $N^{\prime \prime}$ to $N^{\prime \prime}+N-1$. We have $S \subset S^{\prime}$.

We denote by $\boldsymbol{K}$ the $N^{\prime} \times N^{\prime}$ array obtained by zero padding and shifting from the original PSF (for instance the image of a star within the FOV); $\boldsymbol{K}$ is extended periodically outside $S^{\prime}$, with period $N^{\prime}$ in both directions. Then we can introduce the matrix $A$ defined by:

$(A \boldsymbol{f})(\boldsymbol{n})=\sum_{\boldsymbol{n}^{\prime} \in S^{\prime}} \boldsymbol{K}\left(\boldsymbol{n}-\boldsymbol{n}^{\prime}\right) \boldsymbol{f}\left(\boldsymbol{n}^{\prime}\right), \quad \boldsymbol{n} \in S$,

and the corresponding transposed matrix given by:

$\left(A^{T} \boldsymbol{g}\right)\left(\boldsymbol{n}^{\prime}\right)=\sum_{\boldsymbol{n} \in S} \boldsymbol{K}\left(\boldsymbol{n}-\boldsymbol{n}^{\prime}\right) \boldsymbol{g}(\boldsymbol{n}), \quad \boldsymbol{n}^{\prime} \in S^{\prime}$.

We point out that the matrix $A$ transforms $N^{\prime} \times N^{\prime}$ arrays into $N \times N$ arrays while the matrix $A^{T}$ works in the opposite direction.

If we assume that the noise of the image is dominated by photon counting, then it is quite natural to look for the standard Maximum Likelihood (ML) approach to image reconstruction (Shepp \& Vardi 1982; see also Bertero \& Boccacci 1998). The maximization of the log-likelihood function is equivalent to the minimization of the Csiszár I-divergence (Csiszár 1991):

$I(\boldsymbol{f} \mid \boldsymbol{g})=\sum_{\boldsymbol{n} \in \boldsymbol{S}}\left\{\boldsymbol{g}(\boldsymbol{n}) \ln \frac{\boldsymbol{g}(\boldsymbol{n})}{(A \boldsymbol{f})(\boldsymbol{n})}+[(A \boldsymbol{f})(\boldsymbol{n})-\boldsymbol{g}(\boldsymbol{n})]\right\}$.

where, as usual, the quotient of two arrays is defined pixel by pixel.
As it is known, this functional is convex and bounded from below so that all its minima are global. Since our deconvolution problem is under-determined, the functional has several minima (but also in the case of determined problems the uniqueness of the minimum is not proved). Necessary and sufficient conditions for an array $\boldsymbol{f}$ to be a minimum of $I(\boldsymbol{f} \mid \boldsymbol{g})$ in the closed cone of the non-negative arrays are provided by the well-known Kuhn-Tucker conditions (KTC):

$\boldsymbol{f}\left(\boldsymbol{n}^{\prime}\right)\left[\nabla_{\boldsymbol{f}} I(\boldsymbol{f} \mid \boldsymbol{g})\right]\left(\boldsymbol{n}^{\prime}\right)=0, \quad \boldsymbol{n}^{\prime} \in S^{\prime}$,

$\boldsymbol{f}\left(\boldsymbol{n}^{\prime}\right) \geq 0, \quad\left[\nabla_{\boldsymbol{f}} I(\boldsymbol{f} \mid \boldsymbol{g})\right]\left(\boldsymbol{n}^{\prime}\right) \geq 0$

the gradient being given by:

$\left[\nabla_{\boldsymbol{f}} I(\boldsymbol{f} \mid \boldsymbol{g})\right]\left(\boldsymbol{n}^{\prime}\right)=-\left(A^{T} \frac{\boldsymbol{g}}{A \boldsymbol{f}}\right)\left(\boldsymbol{n}^{\prime}\right)+\alpha\left(\boldsymbol{n}^{\prime}\right)$,

where the array $\alpha$ is given by:

$\alpha\left(\boldsymbol{n}^{\prime}\right)=\sum_{n \in S} K\left(\boldsymbol{n}-\boldsymbol{n}^{\prime}\right), \quad \boldsymbol{n}^{\prime} \in S^{\prime}$.

This array is obtained from the sum of the elements of the "columns" of the imaging matrix $A$. If the PSF is normalized to unit volume:

$\sum_{n^{\prime} \in S^{\prime}} \boldsymbol{K}\left(\boldsymbol{n}^{\prime}\right)=1$

then $\alpha\left(\boldsymbol{n}^{\prime}\right)$ is approximately 1 in the central region of the image domain $S$ and becomes less than 1 in pixels close to the boundary of $S$, both inside and outside $S$, approaching zero in pixels far from $S$. The decrease of $\alpha$ depends on the behaviour of the PSF.

If we write explicitly the first KTC we get the equation:

$\alpha \boldsymbol{f}=\boldsymbol{f}\left(A^{T} \frac{\boldsymbol{g}}{A \boldsymbol{f}}\right)$,

where, for simplicity, we do not indicate the dependence on $\boldsymbol{n}^{\prime}$. By iterating this condition, we obtain RLM:

$\boldsymbol{f}^{(k+1)}=\frac{1}{\alpha} \boldsymbol{f}^{(k)}\left(A^{T} \frac{\boldsymbol{g}}{A \boldsymbol{f}^{(k)}}\right)$,

each iterate being defined on $S^{\prime}$.

As it is known, for any initial guess, the iterates converge to a minimum of the functional of Eq. (3) (see, for instance, Lange \& Carson 1984); in order to select one of them, one can use the standard initialization of RLM, namely a constant array. This is the choice we propose for the algorithm described in the following.

We also observe that, by summing with respect to $\boldsymbol{n}^{\prime}$ both sides of Eq. (9), we get:

$\sum_{\boldsymbol{n}^{\prime} \in S^{\prime}} \alpha\left(\boldsymbol{n}^{\prime}\right) \boldsymbol{f}\left(\boldsymbol{n}^{\prime}\right)=\sum_{\boldsymbol{n} \in S} \boldsymbol{g}(\boldsymbol{n})$

and this relation is also true for all the iterates $\boldsymbol{f}^{(k)}$. Its interpretation is the following: the number of photons emitted by the object is greater than the number of photons detected in the FOV, because some of them are not collected within the FOV; the fractions of the outside photons contributing to the FOV are given by the values of the array $\alpha$. 
The algorithm of Eq. (10), provides a reconstruction of the object $f$ defined over the domain $S^{\prime}$, even if it is not obvious that the reconstruction is reliable outside $S$. Moreover the computational cost is high, excessive for large images, because the action of the matrices $A$ and $A^{T}$ can not be computed by means of the FFT. In the following, we propose a trick allowing for a reasonably efficient implementation of the algorithm of Eq. (10) or, more precisely, of an algorithm close to that. Indeed, the reconstruction region will not be a pre-defined square but a domain selected by the PSF: in the previous analysis we considered a square only for illustration purposes.

To get an approach which is independent of the extent of the PSF and may work also in the case where the PSF has different extents in different directions (for instance, the LBT case), we consider $2 N \times 2 N$ arrays, denoted by a bar. They are indexed by a multi-index $\boldsymbol{n}$ defined in such a way that $\{0,0\}$ is the bottomleft pixel. Thus we have a re-indexing of $S$ and $S^{\prime}$ : for instance $\{N / 2, N / 2\}$ is the bottom-left pixel of $S$. We denote by $\bar{S}$ the set of all values of the multi-index; it contains both $S$ and $S^{\prime}$.

The first step is to extend the detected image to $\bar{S}$ by zero padding; we denote by $\overline{\boldsymbol{g}}$ the result. If we assume that also the object is defined on $\bar{S}$, we denote by $\bar{f}$ the corresponding array. Therefore both $\overline{\boldsymbol{f}}$ and $\overline{\boldsymbol{g}}$ have the same extent.

Next we introduce the following matrix $\bar{A}$ :

$$
(\bar{A} \overline{\boldsymbol{f}})(\boldsymbol{n})=\sum_{\boldsymbol{n}^{\prime} \in \bar{S}} \overline{\boldsymbol{K}}\left(\boldsymbol{n}-\boldsymbol{n}^{\prime}\right) \overline{\boldsymbol{f}}\left(\boldsymbol{n}^{\prime}\right), \quad \boldsymbol{n} \in \bar{S},
$$

where the PSF $\overline{\boldsymbol{K}}$, defined on $\bar{S}$, is obtained by zero padding and shifting from the original PSF, and is extended periodically outside $\bar{S}$, with period $2 N$ in both directions. This matrix and its transposed are block-circulant and therefore their action can be computed by means of the FFT, at the cost of a $2 N \times 2 N$ cyclic convolution.

We consider now the functional of Eq. (3) with $\boldsymbol{f}, \boldsymbol{g}, A$ and $S$ replaced respectively by $\overline{\boldsymbol{f}}, \overline{\boldsymbol{g}}, \bar{A}$ and $\bar{S}$. As usual, we consider $x \ln x=0$ if $x=0$, so that the sum is extended, in practice, to $S$. However, if we keep also the zero values of $\bar{g}$, then the first KTC, can be written as follows:

$\bar{\alpha} \overline{\boldsymbol{f}}=\overline{\boldsymbol{f}}\left(\bar{A}^{T} \frac{\overline{\boldsymbol{g}}}{\bar{A} \overline{\boldsymbol{f}}}\right)$,

the array $\bar{\alpha}$ being defined on $\bar{S}$ by:

$\bar{\alpha}\left(\boldsymbol{n}^{\prime}\right)=\sum_{\boldsymbol{n} \in S} \overline{\boldsymbol{K}}\left(\boldsymbol{n}-\boldsymbol{n}^{\prime}\right), \quad \boldsymbol{n}^{\prime} \in \bar{S}$.

$\bar{\alpha}$ coincides with $\alpha$ on $S^{\prime}$; moreover, if we denote by $\boldsymbol{M}_{S}(\boldsymbol{n})$ the mask of the original image domain, i. e. the array which is 1 when $\boldsymbol{n} \in S$ and 0 elsewhere, then we get:

$\bar{\alpha}\left(\boldsymbol{n}^{\prime}\right)=\left(A^{T} \boldsymbol{M}_{S}\right)\left(\boldsymbol{n}^{\prime}\right), \quad \boldsymbol{n}^{\prime} \in \bar{S}$,

and therefore $\bar{\alpha}$ can be efficiently computed by means of the FFT.

We point out that $\bar{\alpha}$ may contain zeros or very small values. Then, from Eq. (13), it follows that the corresponding values of $\bar{f}$ are also zero or very small. Moreover, we observe that, if $\bar{f}$, defined on $\bar{S}$, is zero in $\bar{S}-S^{\prime}$, we have $\bar{A} \bar{f}=A \boldsymbol{f}$ in $S$ and therefore the quotient $\bar{g} / \bar{A} \boldsymbol{f}$ coincides with the quotient $\boldsymbol{g} / A \boldsymbol{f}$ on $S$ and is zero outside. If we take into account this property, it is easy to understand that, by applying $\bar{A}^{T}$ to this quotient, we obtain:

$\left(\bar{A}^{T} \frac{\overline{\boldsymbol{g}}}{\bar{A} \overline{\boldsymbol{f}}}\right)\left(\boldsymbol{n}^{\prime}\right)=\left(A^{T} \frac{\boldsymbol{g}}{A \boldsymbol{f}}\right)\left(\boldsymbol{n}^{\prime}\right), \quad \boldsymbol{n}^{\prime} \in S^{\prime}$.

This relation between quantities with and without bar can be used to understand the relationship between the algorithm we propose and the algorithm of Eq. (10).

In order to write RLM by iterating Eq. (13) and avoid division by zero, we must constrain the iterates $\overline{\boldsymbol{f}}^{(k)}$ to be zero outside $S^{\prime}$, or outside a set replacing conveniently $S^{\prime}$. To this purpose we introduce a threshold $\sigma<1$ and we consider the set $S_{\sigma}^{\prime}$ where $\bar{\alpha}>\sigma$ (we can take, for instance, $\sigma=10^{-2}$ or even smaller): the pixels outside this set do not contribute significantly to the detected image and therefore they can be forgotten. This result can be obtained by means of the following window:

$\overline{\boldsymbol{w}}\left(\boldsymbol{n}^{\prime}\right)= \begin{cases}\frac{1}{\bar{\alpha}\left(\boldsymbol{n}^{\prime}\right)}, & \text { if } \bar{\alpha}\left(\boldsymbol{n}^{\prime}\right)>\sigma, \\ 0, & \text { otherwise, }\end{cases}$

which can be inserted in the RLM algorithm. The result is:

$\overline{\boldsymbol{f}}^{(k+1)}=\overline{\boldsymbol{w}} \overline{\boldsymbol{f}}^{(k)}\left(\bar{A}^{T} \frac{\overline{\boldsymbol{g}}}{\bar{A} \overline{\boldsymbol{f}}^{(k)}}\right)$,

and all the iterates are automatically zero outside $S_{\sigma}^{\prime}$. Moreover, in order to extract one specific minimum of $I(\boldsymbol{f} \mid \boldsymbol{g})$, we take as a rule to initialize the iterations with a constant array.

Thanks to Eq. (16) we can conclude that each iterate $\bar{f}^{(k)}$ is close to the $\boldsymbol{f}^{(k)}$ of Eq. (10); more precisely they coincide if the $\boldsymbol{f}^{(k)}$ are the iterates corresponding to the problem with $S^{\prime}$ replaced by $S_{\sigma}^{\prime}$. We also remark that the iterates $\overline{\boldsymbol{f}}^{(k)}$ satisfy a flux condition similar to that of Eq. (11), again with $S^{\prime}$ replaced by $S_{\sigma}^{\prime}$.

A minor modification of the algorithm consists in taking into account the background, due to diffuse emission from the sky and the detector (Snyder et al. 1993). With this modification the constraint of non-negativity, which is implicit in RLM, becomes active. If we assume that the background is constant over the image domain, with a pixel-value $b$, and we denote by $\boldsymbol{b}$ the corresponding constant array, then the modification of the RLM algorithm of Eq. (18), is as follows:

$\overline{\boldsymbol{f}}^{(k+1)}=\overline{\boldsymbol{w}} \overline{\boldsymbol{f}}^{(k)}\left(\bar{A}^{T} \frac{\overline{\boldsymbol{g}}}{\bar{A} \overline{\boldsymbol{f}}^{(k)}+\boldsymbol{b}}\right)$.

In such a case, the flux of the iterates is not given by Eq. (11), which applies to the case of zero background, but it satisfies approximately the following condition:

$\sum_{\boldsymbol{n}^{\prime} \in S_{\sigma}^{\prime}} \bar{\alpha}\left(\boldsymbol{n}^{\prime}\right) \overline{\boldsymbol{f}}^{(k)}\left(\boldsymbol{n}^{\prime}\right)=\sum_{\boldsymbol{n} \in S}\{\boldsymbol{g}(\boldsymbol{n})-b\}$.

It may be useful to normalize the result of each iteration in such a way that this condition is satisfied (Lanteri et al. 2002). This normalization is introduced in the RL-like algorithms described and validated in Anconelli et al. (2005). 


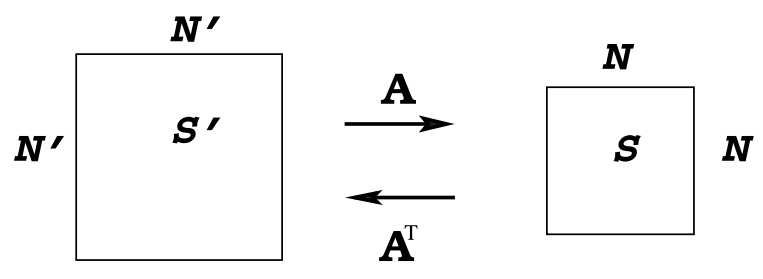

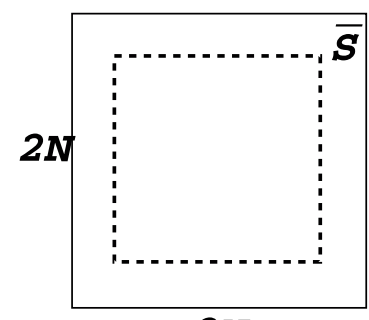

\section{$2 N$}

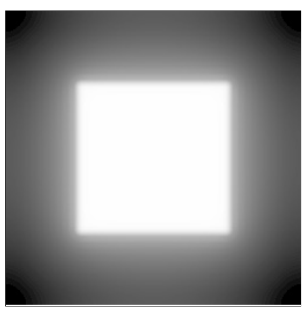

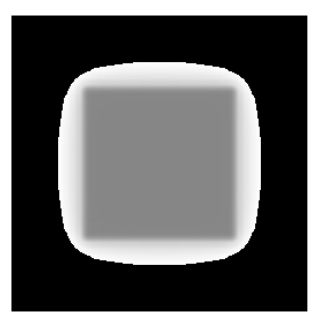

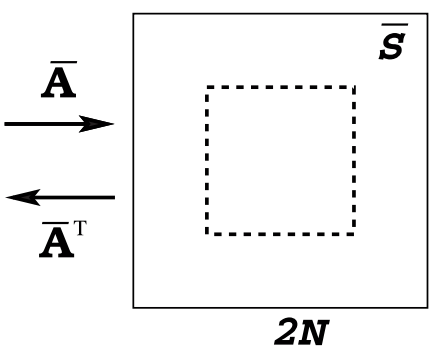

Fig. 1. Pictorial description of the method proposed in this paper: in the upper panels we have the initial formulation of the problem while in the middle panels we have the situation obtained by extending the arrays by zero-padding; finally, in the bottom-left panel we give the array $\bar{\alpha}$ (log-scale representation) in the case of the PSF of Fig. 2 and in the bottom-right panel the window $\overline{\boldsymbol{w}}$ (linear-scale representation), corresponding to $\sigma=10^{-2}$. The support of this window is close to the array $N^{\prime} \times N^{\prime}$.

In conclusion, the method consists of the following steps:

- define $\overline{\boldsymbol{g}}$ by zero padding of the detected image $\boldsymbol{g}$;

- compute $\bar{\alpha}$ defined in Eq. (15);

- compute the window $\overline{\boldsymbol{w}}$ defined in Eq. (17);

- initialize the algorithm with $f^{(0)}=$ const.;

- given $\overline{\boldsymbol{f}}^{(k)}$ compute $\overline{\boldsymbol{f}}^{(k+1)}$ by means of Eq. (19);

- stop the iterations according to a pre-assigned criterion.

This algorithm has been implemented in IDL and will be inserted in the software package AIRY (Correia et al. 2002). A pictorial representation of the method is given in Fig. 1. It is obvious that, even if for explanatory reasons we placed the $N \times N$ image at the center of the $2 N \times 2 N$ array, thanks to the periodicity, implicit in the use of the FFT, it can be placed in any part of this array. In particular, for specific applications, it may be convenient to put it in one of the four quadrants.

\section{Numerical examples}

We have tested the method described above on several simulated data, and we have always obtained excellent results. We illustrate these results by means of two specific examples.

First we consider a test object already used in our previous papers (Carbillet et al. 2002): it is an embedded young stellar

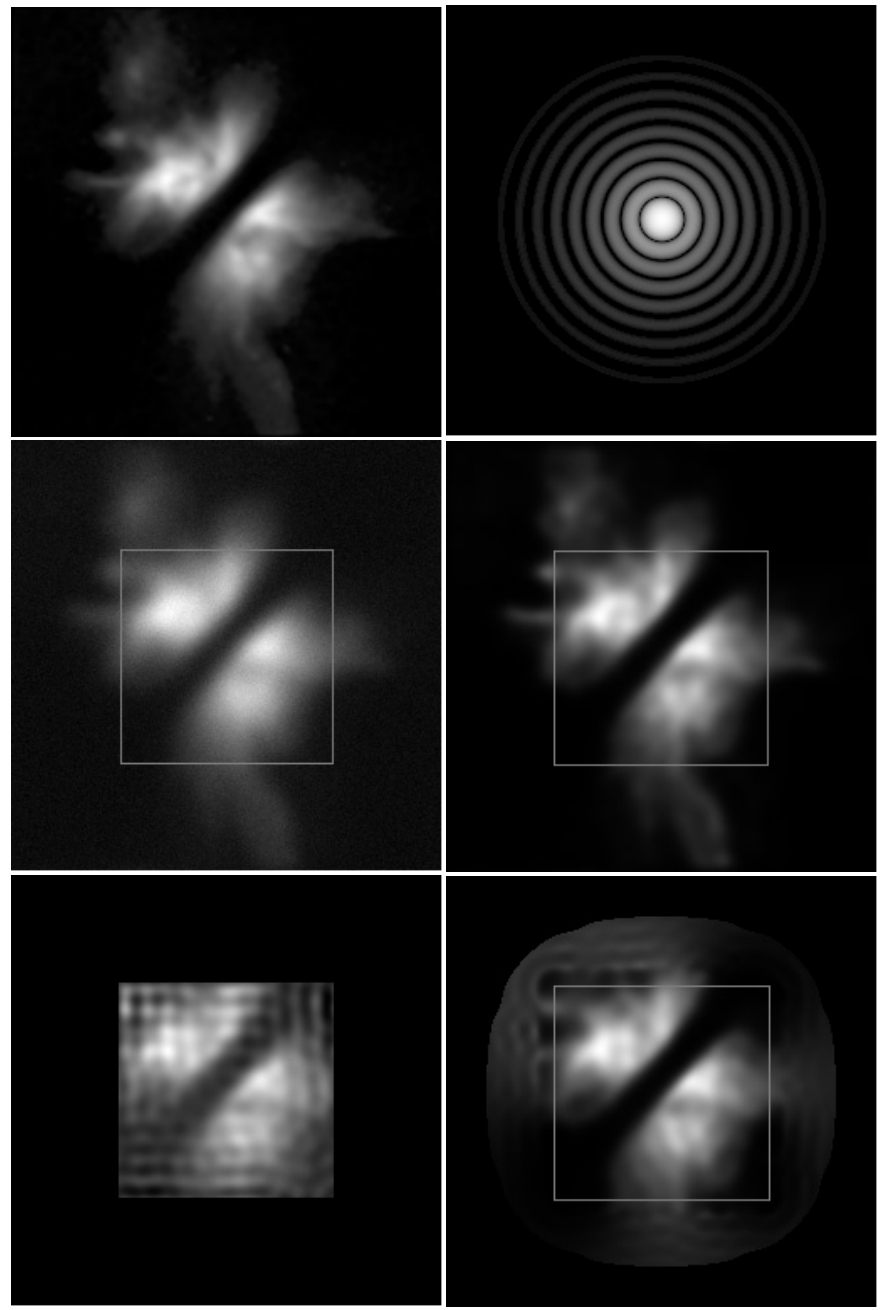

Fig. 2. Upper panels: the object (left) and the PSF (right-log-scale representation). Middle panels: the image obtained by convolving the object with the PSF and perturbing the result with Poisson noise (left); the reconstruction of the complete image by means of RLM (right). Lower panels: the reconstruction of the central part of the image obtained by means of the standard RLM $(l e f t)$; reconstruction of the same part by means of the method described in this paper (right).

object, also known as Class I object, derived from HST nearinfrared observations of IRAS $04302+2247$. This is a remarkable object with a butterfly shape. The corresponding array is $256 \times 256$. Just to fix some astronomical parameters, we generated the image by assuming observation in $K$ band with a perfect mirror and no atmosphere, so that the PSF is given by the Airy function; moreover we assumed: integrated magnitude of the object 15; ideal PSF with a FWHM of about 6 pixels; integration time of $1200 s$; efficiency of the telescope 0.4. The ideal image obtained by convolving the object with the PSF has been perturbed with Poisson noise. In Fig. 2 we show the object, the PSF (log-scale) and the image. This image has been deconvolved by means of standard RLM and the result is also shown in Fig. 2. The accuracy of this restoration is quite good, with an integrated rms error smaller than $1 \%$. The number of iterations required for getting this result is of the order of 600 .

Next, we extracted from the center of the image an array $128 \times 128$ (the square with white boundaries indicated in Fig. 2). 

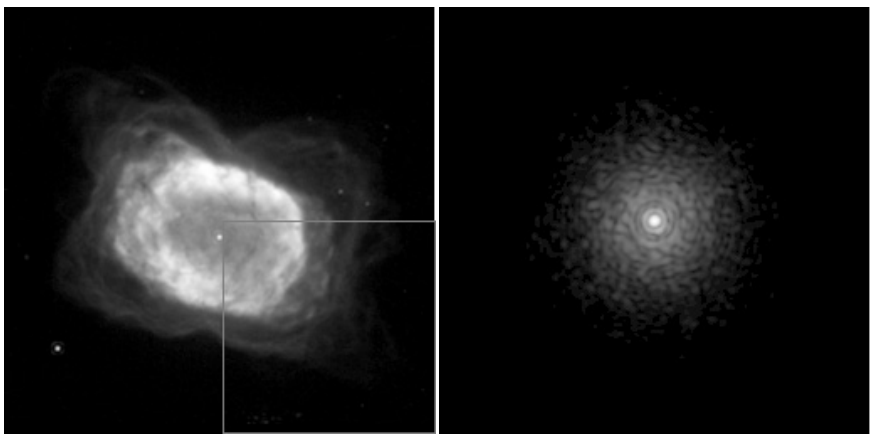

Fig. 3. Example of application with a non-symmetric PSF. Left panel: the object, HST image of the nebula NGC7027; the square with white boundaries indicate the quadrant used for testing our method. Right panel: the AO-corrected PSF used for generating the image.

If this image is deconvolved by means of the standard RLM, the result is strongly corrupted by ripples, as it is obvious. This result is shown in the lower-left panel of Fig. 2. On the other hand, if we use the method proposed in this paper, the result is that shown in the lower-right panel. This result needs a few comments. It is clear that the reconstruction outside the image domain is not good, even if it is not so bad in a border which is about six pixels wide (the extent of the central lobe of the PSF). However, if we extract the reconstructed image corresponding to the image domain and we compare with that obtained from the global deconvolution (middle-right panel of Fig. 2), the integrated rms difference is of the order of $2 \%$.

In the second example the object is the HST image of the nebula NGC7027 and the PSF takes into account Adaptive Optics (AO) corrections, with a Strehl Ratio (SR) of about $52 \%$. The object and the PSF are shown in Fig. 3. The image (not shown) has been generated by convolving the object with the PSF and using for the other parameters the same values of the previous example. The full image has been deconvolved with the same PSF and the minimum restoration error is reached after 100 iterations. The accuracy is quite good since the integrated rms error is about $0.15 \%$. Next we extracted from the image the lower-right quadrant and we applied our method. We got convergence after 75 iterations, with an integrated rms error of about $0.3 \%$. The difference between the two reconstructions is not visible, as shown in Fig. 4. We obtain similar results using a PSF with $S R=38 \%$, hence even more asymmetric than that of Fig. 3.

Finally we investigated the possibility of using this approach for the block-deconvolution of an extended image, which is relevant in the case of space-variant PSFs. Indeed, some of the proposed methods (Fish et al. 1996; Aubailly et al. 2004) consist in partitioning the FOV in partly overlapping subdomains to reduce boundary effects in their central parts. Then, the full deconvolved image is obtained as a mosaic of the central parts. Since our method strongly reduces the boundary effects, one can argue that it is possible to reduce the overlapping of the sub-domains. To verify this possibility, we partitioned the image of the object of Fig. 3 into four non-overlapping parts corresponding to the four quadrants, and the four $128 \times 128 \mathrm{im}-$ ages were deconvolved by means of our method. Next the full
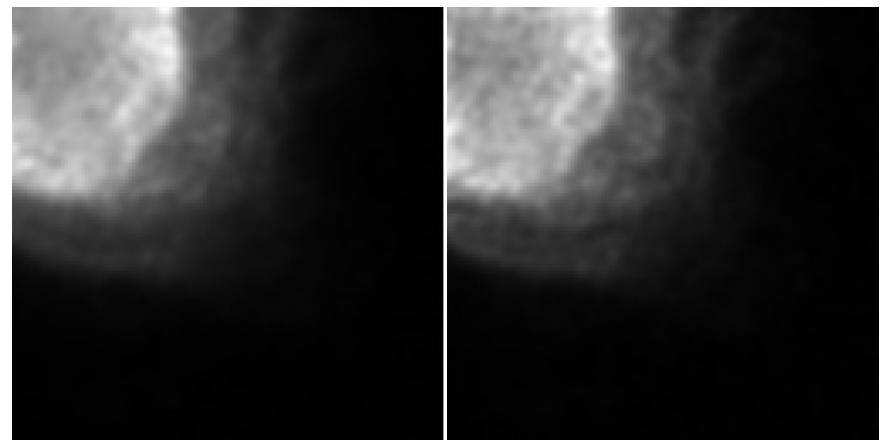

Fig. 4. Example of application with a non-symmetric PSF. Left panel: the reconstruction of the lower-right quadrant of the object of Fig. 3, as obtained from the reconstruction of the full image. Right panel: the reconstruction of the same quadrant obtained with the method presented in this paper.

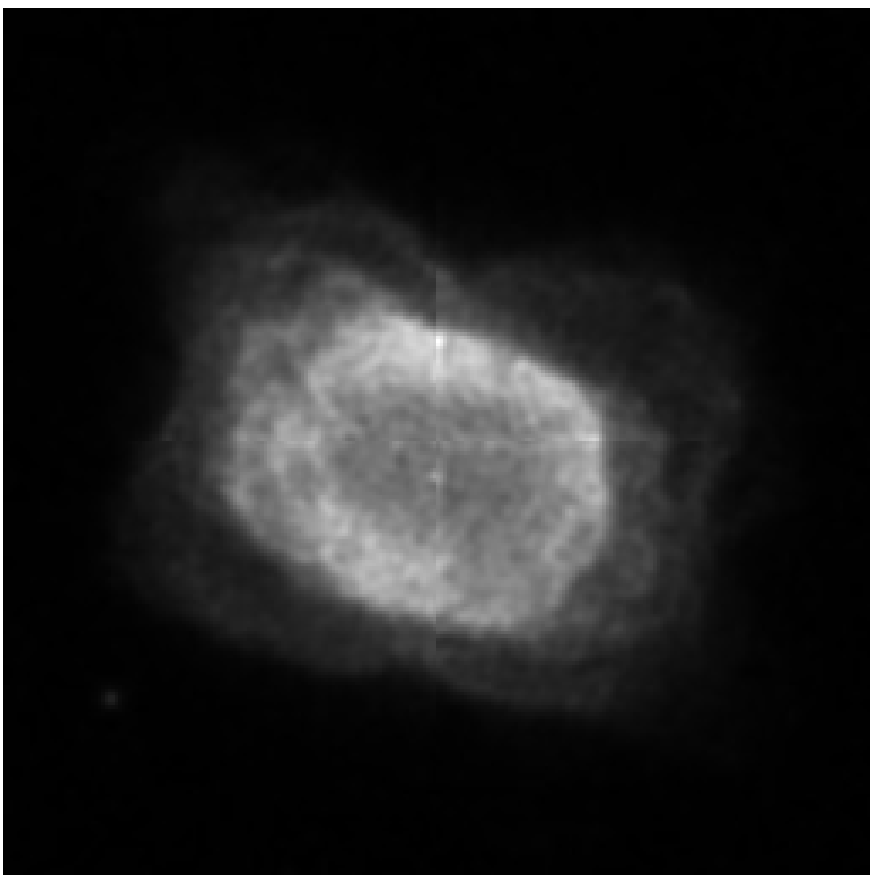

Fig. 5. Example of the application of the method to the blockdeconvolution of an image: reconstruction of the object of Fig. 3 by deconvolving separately the four quadrants of its image by means of the method proposed in this paper.

image is obtained as a mosaic of the four reconstructions obtained in the four quadrants. The result is shown in Fig. 5. The effect of the mosaicking is visible but it is restricted to a few pixels around the boundaries of the four quadrants. Therefore we think that this result is quite promising and deserves further investigation.

\section{Concluding remarks}

The method described in this paper for the reduction of boundary effects in image deconvolution is based on a suitable implementation of RLM applied to an under-determined problem. The increase of the computational cost, with respect to the direct application of RLM to the detected image, is reasonable. The idea consists in using RLM for reconstructing the 
astronomical object in a domain which is broader than the FOV. The reconstruction outside the FOV is not reliable but that within the FOV has essentially the same accuracy of the reconstruction obtained from the complete image of the target.

The numerical simulations discussed in the paper are performed in the case of the so-called inverse crime: the same PSF is used for convolving and deconvolving the astronomical target. The assumption is not realistic in the case, for instance, of ground-based telescopes equipped with an $\mathrm{AO}$ system. If the correction over the FOV is not uniform, then the PSF in the region of the target does not coincide with the PSF obtained by observing a reference star. Therefore the robustness of the method with respect to inaccurate knowledge of the PSF must be checked.

This is one of the research lines which can be considered starting from the idea presented in this paper. For instance the approach must be extended to the deconvolution of images of Fizeau interferometers such as the already mentioned LBT. Since the expected FOV of the detector of LINC-NIRVANA, the German-Italian beam combiner for LBT, is about $10^{\prime \prime} \times 10^{\prime \prime}$, a complex and extended object may not be completely contained within this FOV. Hence the extension of the method to the deconvolution of multiple interferometric images of the same target is potentially very useful.

Another possible application is the reconstruction of extended images in the case of space-variant PSFs, both for monolithic mirrors and Fizeau interferometers. One approach used in Astronomy (Aubailly et al. 2004) consists in partitioning the FOV in sub-domains where the PSF is approximately space invariant and in using a standard deconvolution method such as RL. Moreover, partly overlapping sub-domains are considered for reducing boundary effects in their central parts. The reconstructed image is obtained as a mosaic of these central parts. Since our deconvolution method is essentially free of boundary artifacts, then it is possible to consider a partition of the FOV into slightly overlapping sub-domains, with a gain in the number of domains used for a complete reconstruction of the target and an improvement of the accuracy.

Acknowledgements. We thanks the referee for many valuable remarks which significantly contributed to the improvement of the paper.

\section{References}

Aubailly M., Roggemann M. C., \& Schulz T. 2004, Proc. 2004, AMOS Technical Conf., 131

Anconelli B., Bertero M., Boccacci P., Lanteri H., \& Carbillet M. 2005, A\&A, 430, 731

Bertero M., \& Boccacci P. 1998, Introduction to Inverse Problems in Imaging (Bristol: IOP Publishing)

Carbillet M., Correia S., Boccacci P., \& Bertero M. 2002, A\&A, 387, 744

Chan R., Ng M., \& Tang W. C. 1999, SIAM J. Sci. Comput., 21, 851

Correia S., Carbillet M., Boccacci P., Bertero M., \& Fini L. 2002, A\&A, 387, 733

Csiszár I. 1991, Ann. Stat., 19, 2032

Fish D. A., Grochmalicki J., \& Pike E. R. 1996, OSAJ, 13, 464

Lange K., \& Carson R. 1984, J. Comp. Ass. Tom., 8, 306

Lanteri H., Roche M., \& Aime C. 2002, Inverse Problems, 18, 1397

Serra Capizzano S. 2003, SIAM J. Sci. Comput., 25, 1307

Shepp L. A., \& Vardi Y. 1982, IEEE Trans. Med. Im., 1, 113

Snyder D. L., Hammoud A. M., \& White R. L. 1993, JOSA, A10, 1014 